\section{Consent insufficient for data release}

In their Policy Forum "Toward unrestricted use of public genomic data" (25 January, p. 350), R. I. Amann et al. argue that once data has been cleared for release to the public domain by institutions, it should be open for use without further restrictions. However, they neglect the key point that researchers and their institutions are entrusted by research participants, funders, and others with weighing the pros and cons of public data release. By suggesting that informed consent can provide a straightforward path to data release, they overlook evidence that once people understand their options, only a little more than half opt for open data sharing, and some refuse data sharing altogether $(1,2)$.

This evidence further shows that some research participants have concerns that uses of their data might not fit with their norms or values or might disadvantage certain populations. Despite their openness to wide use, they do not think ethics review and informed consent is sufficient to remove restrictions on the release of sensitive human data. Simply put, informed consent is a necessary, but far from sufficient condition for data sharing $(3,4)$.

Equally important, Amann et al.'s proposal for data sharing through open access databases does not reflect funders' policies. Rather, funders expect and, in some cases, mandate that researchers adopt specific organizational measures to safeguard personal data. For example, the National Institutes of Health (NIH) policy on Genomic Data Sharing explicitly requires that data generators develop genomic data sharing plans and data users submit their requests to data access committees for review (5). The imperative for adequate data governance has also been stressed by other major funding agencies, such as the Wellcome Trust Expert Advisory Group on Data Access (6).

Admittedly, poorly designed regulation can stifle legitimate genomic data sharing that promotes the public good. Regulatory frameworks do, however, serve critical purposes, including ensuring consideration of the intricate ethical, legal, social, and political concerns inherent in many aspects of science, including genomics. Amann et al.'s uncritical use of the notion of "openness" suggests that once data has been made open, its use is unaffected by structural issues such as the unequal distribution of power and influence. This is particularly problematic in cases of for-profit enterprises that are not accountable to the public (7). Although Amann et al.'s suggestions seem emancipatory and respectful of ethical concerns, their proposal overlooks the wider political economy in which data use is embedded and conflates ethics with a rather formulaic adherence to legal and institutional guidelines and consent forms.

This complex challenge cannot be solved with a single model for data sharing governance. The currently favored model of controlled-access data sharing adopted by the $\mathrm{NIH}$ and others is far from perfect (8). Better alternatives are emerging, such as the Global Alliance for Genomics Health's Beacon federated model for data sharing (9). We need approaches to data sharing that address, on a case by case basis, how public release of data affects distribution of burdens and benefits across and within populations.

Dianne Nicol ${ }^{1 *}$, Lisa Eckstein ${ }^{1}$, Heidi Beate Bentzen 2,3, Pascal Borry 4 , Mike Burgess 5 , Wylie Burke $^{6}$, Don Chalmers ${ }^{1}$, Mildred Cho ${ }^{7}$, Edward Dove $^{8}$, Stephanie Fullerton ${ }^{6}$, Ryuchi Ida ${ }^{9}$, Kazuto Kato ${ }^{10}$, Jane Kaye ${ }^{11}$, Barbara Koenig ${ }^{12}$, Spero Manson ${ }^{13}$, Kimberlyn McGrail ${ }^{14}$, Amy McGuire ${ }^{15}$, Eric Meslin ${ }^{16}$, Kieran O'Doherty ${ }^{17}$, Barbara Prainsack ${ }^{18}$, Mahsa Shabani ${ }^{4}$, Holly Tabor $^{7}$, Adrian Thorogood ${ }^{19}$, Jantina de Vries 20

${ }^{1}$ Law Faculty, University of Tasmania, Hobart, Tasmania, 7001, Australia. ${ }^{2}$ Norwegian Research Center for Computers and Law, Faculty of Law, University of Oslo, 0130 Oslo, Norway. ${ }^{3}$ Centre for Medical Ethics, Faculty of Medicine, University of Oslo, 0318 Oslo, Norway. ${ }^{4}$ Centre for Biomedical Ethics and Law, University of Leuven, 3000 Leuven, Belgium. ${ }^{5}$ School of Population Health, University of British Columbia, Vancouver, BC, V6T 1Z3, Canada. ${ }^{6}$ School of Medicine, University of Washington, Seattle, WA 98195, USA ${ }^{7}$ Centre for Biomedical Ethics, Stanford Medicine, Stanford, CA 94305, USA. ${ }^{8}$ School of Law, University of Edinburgh, Edinburgh EH8 9YL, UK. ${ }^{9}$ Kyoto University, Kyoto, $606-8501$, Japan. ${ }^{10} \mathrm{Graduate}$ School of Medicine, Osaka University, 565-0871, Japan. ${ }^{11}$ Centre for Health, Law, and Emergent Technologies, University of Oxford, Oxford, UK. ${ }^{12}$ School of Nursing, University of California, San Francisco, San Francisco, CA xxxxxx, USA. ${ }^{13}$ Colorado School of Public Health, University of Colorado, Aurora, CO 80045, USA. ${ }^{14}$ Centre for Health

Services and Policy Research, University of British Columbia, Vancouver, BC, V6T 1Z3, Canada. ${ }^{15}$ Baylor College of Medicine, Houston, TX 77030, USA. ${ }^{16}$ Council of Canadian Academies, Ottawa, ON, K2P 2K3, Canada.

${ }^{17}$ Department of Psychology, University of Guelph, Guelph, N1G 2WY, Canada. ${ }^{18}$ Department of Political Science, University of Vienna, 1140, Vienna, Austria. ${ }^{19}$ Centre of Genomics Policy, McGill University, Montreal, QC, H3A 0G1, Canada. ${ }^{20}$ Department of Medicine University of Cape Town, Cape Town, 7791, South Africa. *Corresponding author. Email: dianne.nicol@utas.edu.au

All authors sign this letter in their personal capacities, not as spokespersons for any institution or organization with which they are affiliated.

\section{REFERENCES AND NOTES}

1. A. L. McGuire et al., Genet. Med. 13, 948 (2011).

2. N. Shah et al., Eur. J. Hum. Genet. 10.1038/s41431019-0344-2 (2019).

3. J. Teng et al., Intl. J. Pop. Data Sci. 3, 364 (2018).

4. R. E. McWhirter et al., J. Pers. Med. 4, 459 (2014).

5. NIH, NIH GDS Policies (https://osp.od.nih.gov/scientific-sharing/policies/).

6. Expert Advisory Group on Data Access, "Governance of data access" (2015); https://wellcome.ac.uk/sites/default/files/governance-ofdata-access-eagda-jun15.pdf.

7. S. Zuboff, The Age of Surveillance Capitalism: The Fight for the Future at the New Frontier of Power (PublicAffairs, 2019).

8. D Mascalzoni et al., Ann. Intern. Med. 170, 332 (2019).
9. The Global Alliance for Genomics and Health, Science 352, 1278 (2016) 